\title{
Optimal Policies of Social Infrastructures Maintenance using Shock and Damage Model
}

\author{
Akihiro Yamane \\ Department of Engineering, Tottori University, \\ Minami 4-101, Koyama, Tottori-Shi, Tottori, 680-8552, Japan. \\ Corresponding author: M20J6021Z@edu.tottori-u.ac.jp \\ Kodo Ito \\ Department of Engineering, Tottori University, \\ Minami 4-101, Koyama, Tottori-Shi, Tottori, 680-8552, Japan. \\ E-mail: itokodo@tottori-u.ac.jp

\section{Yoshiyuki Higuchi} \\ Faculty of Symbiotic Systems Science, Fukushima University, \\ Fukushima-Shi Fukushima, Japan. \\ E-mail: higuchi@sss.fukushima-u.ac.jp \\ (Received on February 10, 2021; Accepted on October 25, 2021)
}

\begin{abstract}
Social infrastructures such as roads and bridges are indispensable for our lives. They have to be maintained continuously and such maintenance has become a big issue in Japan. Social infrastructures are maintained under strict restrictions such as decreasing in local finance revenue and scarcity of skilful engineers. Various kinds of factors such as inspection periods, maintenance costs, and degradation levels, are necessary to consider in establishing efficient maintenance plans of social infrastructures. Furthermore, the special circumstances of social infrastructures such as the delay of constructions which is caused by the scarcity of budget, must be discussed for the efficient maintenance plan. For such discussion, the stochastic cost model which contains preventive and corrective maintenances is useful. Although these models have been studied in mechanical and electronic systems, unique characteristics of social infrastructures such as their enormous scale and delays due to maintenance budget restrictions must be considered when such social infrastructure models are discussed. In this paper, we establish maintenance models of infrastructures which some of preventive maintenance must be prolonged. The expected maintenance cost rate is established using the cumulative damage model and optimal policies which minimizes them are considered. Three basic models and their extended models which consider natural disasters are discussed.
\end{abstract}

Keywords- Social infrastructures, Asset management, Maintenance, Optimal policy, Cumulative damage model.

\section{Introduction}

Social infrastructures such as roads and bridges must maintain high reliability. On the other hand, the national and local governments have various restrictions such as a decrease in budget and technicians, and it is necessary to carry out efficient conservation such as minimizing the cost of conservation. The maintenance of social infrastructures has become a big national issue in 2023 because $39 \%$ of road bridges and $27 \%$ of tunnels in Japan are to exceed 50 years since their construction (Ministry of Land, Infrastructure, Transport and Tourism, 2017). Such budget restrictions cause delays in preventive maintenance (PM) of social infrastructures. When there are multiple social infrastructures which are needed to undergo the PM, the selection which to delay and which to prioritize is a quite difficult issue because multiple factors such as the frequency of infrastructure use and the risk of procrastination must be considered. Furthermore, prioritizing 
between multiple facilities is the quite difficult issue because the scale of social infrastructure is huge, and the maintenance cost varies case by case depending on the degree of deterioration. For undergoing such consideration, the stochastic cost model which contains PM and corrective maintenances (CM) is needed. Although these models have been studied in mechanical and electronic systems, unique characteristics of social infrastructures such as their enormous scale and delays due to maintenance budget restrictions must be considered when such models are considered.

Maintenances are divided into PM and CM (Nakagawa, 2007). Establishing effective PM is important and there exist lots of studies concerning the social infrastructures maintenance plan in the past. The tunnel structure degradation model using Poisson process was recommended by Sakai et al. (2014). Kaito et al. (2005) showed that the Life Cycle Cost of bridge maintenance can be divided into fixed costs and variable costs (Kaito et al., 2005). Obama et al. (2012) discussed a methodology to design optimal scrapping and maintenance policies of infrastructures which are to be crashed due to the demand reduction or the advance of degradation (Obama et al., 2012). Kobayashi et al. (2012) considered the pavement degradation process of both the load surface integrity and the load carrying capacity using the combined Markov deterioration model, and suggested the optimal repair and improve model for minimizing the expected LCC (Kobayashi et al., 2012). Tsuda et al. (2015) represented a methodology for predicting bridge component degradation by using the Markovian transition probability model (Tsuda et al., 2005). Ito et al. (2018) proposed optimal policies of social infrastructure maintenance using Markov Chains (Ito et al., 2018).

The deterioration hazard model is useful for establishing effective maintenance policies because complex deterioration processes can be set in the form of hazard functions and optimal policies can be mathematically discussed. So, such models for road pavement (Kobayashi et al., 2013; Shin and Madanat, 2003) and bridges (Tsuda et al., 2006) was considered. Because the maintenance of social infrastructures is performed annually by local governments and budgets of these local governments are restricted, all of necessary PM cannot be performed by the end of the year and some of them are postponed to the next year. Ito et al. (2018) studied social infrastructure hazard models considering such delayed maintenance (Ito and Nakagawa, 2018) and Kishida et al. (2019) expanded it considering natural disasters (Kishida et al., 2019). In the model, the PM is performed when the cumulative damage surpasses the prespecified managerial level $Z$ and the $C M$ is undergone when the cumulative damage surpasses the prespecified failure level $K(>Z)$. In practice, there exist some damage levels between $Z$ and $K$ because the scale of social infrastructures is huge, and PM costs change depends on the levels. When we consider the damage level of delayed maintenance, we should consider those multiple damage levels.

In this paper, we discuss the social infrastructure maintenance model which has several damage levels. PM costs vary when these damage levels vary, and the PM time is exceeded when the damage level is lower than a certain damage level. The expected cost rate is established and the optimal damage level which minimizes the expected cost rate is discussed. Model 1 is a basic multistage model and Model 3 is an extended model which considers the maintenance delay. Because deriving the formula of Model 3 directly from Model 1 is desperate, a particular case of Model 3 was considered as Model 2 and the mathematical formula form was clarified by the analysis of Model 2. Model 4, 5, and 6 are extensions of Model 1, 2, and 3 in consideration of natural disasters. 


\section{Model 1}

First, we consider the fundamental model (Kishida et al., 2020). Following maintenance policies of infrastructures are assumed:

(i) The social infrastructure is deteriorated with traffics, and its amount of damage is proportional to time $j T(j=1,2, \cdots)$ for a specified $T>0$. An amount $W_{j}$ of damage due to the $j$ th shock has an identical distribution $G(x) \equiv \mathrm{P}_{\mathrm{r}}\left\{W_{j} \leq x\right\}$ with mean time $1 / \mu$.

(ii) The infrastructure damage levels are denoted as discrete levels $Z_{i}(i=1,2, \cdots, K-1, K)$, where $Z_{1}<Z_{2}<\ldots<Z_{K}$ for $K \geq 2$. The $C M$ is undergone at time $j T$ when the cumulative damage surpasses a threshold level $Z_{K}$ at time $j T$. When the cumulative damage is between $Z_{1}\left(<Z_{K}\right)$ and $Z_{K}$, the PM is undergone at time $j T$.

(iii) When the cumulative damage is between $Z i$ and $Z i+1(i=1,2, \cdots, K-1), \mathrm{CM} \operatorname{cost}$ is $c_{K}$ and PM cost is $c_{i}\left(<c_{i+1}\right)$.

The probability which $\mathrm{CM}$ is undergone at time $(j+1) T$ is:

$\sum_{j=0}^{\infty} \int_{0}^{Z_{1}} \bar{G}\left(Z_{K}-x\right) \mathrm{d} G^{(j)}(x)$

where, $\phi^{(j)}(x)(j=1,2, \cdots)$ denotes the $j$-fold Stieltjes convolution of distribution $\phi(x)$,

$\phi^{(0)}(x) \equiv 1$. The probabilities which PM is undergone at time $(j+1) T$ are;

$\sum_{j=0}^{\infty} \int_{0}^{Z_{1}}\left[G\left(Z_{i+1}-x\right)-G\left(Z_{i}-x\right)\right] \mathrm{d} G^{(j)}(x) \quad(i=1,2, \cdots, K-1)$

The mean time to maintenance is:

$\sum_{j=0}^{\infty}(j+1) T \int_{0}^{Z_{1}} \bar{G}\left(Z_{K}-x\right) \mathrm{d} G^{(j)}(x)+\sum_{j=0}^{\infty}(j+1) T \int_{0}^{Z_{1}}\left[G\left(Z_{i+1}-x\right)-G\left(Z_{i}-x\right)\right] \mathrm{d} G^{(j)}(x)$
$=T\left[1+M_{G}\left(Z_{1}\right)\right]$

where, $M_{G}(x) \equiv \sum_{j=1}^{\infty} G^{(j)}(x)$. The total expected cost until maintenance is:

$$
\begin{aligned}
& c_{K} \sum_{j=0}^{\infty} \int_{0}^{Z_{1}} \bar{G}\left(Z_{K}-x\right) \mathrm{d} G^{(j)}(x)+\sum_{i=1}^{K-1} c_{i} \sum_{j=0}^{\infty} \int_{0}^{Z_{1}}\left[G\left(Z_{i+1}-x\right)-G\left(Z_{i}-x\right)\right] \mathrm{d} G^{(j)}(x) \\
& =c_{K}-\sum_{j=2}^{K}\left(c_{j}-c_{j-1}\right)\left[G\left(Z_{j}\right)-\int_{0}^{Z_{1}} \bar{G}\left(Z_{j}-x\right) \mathrm{d} M_{G}(x)\right]
\end{aligned}
$$

Thus, the expected cost rate is, from (3) and (4),

$$
T C_{1}\left(Z_{1}\right)=\frac{c_{K}-\sum_{j=2}^{K}\left(c_{j}-c_{j-1}\right)\left[G\left(Z_{j}\right)-\int_{0}^{Z_{1}} \bar{G}\left(Z_{j}-x\right) d M_{G}(x)\right]}{1+M_{G}\left(Z_{1}\right)}
$$


$T C_{1}\left(Z_{1}\right)$ is expressed as a function of $Z_{l}$ because $\mathrm{PM}$ is undergone when degradation level is greater than $Z_{1}$. We seek an optimal $Z_{1}^{*}$ which minimizes $C_{1}\left(Z_{1}\right)$. Differentiating $C_{1}\left(Z_{1}\right)$ with respect to $Z_{1}$ and setting it equal to zero;

$\sum_{j=2}^{K}\left(c_{j}-c_{j-1}\right) \int_{Z_{j}-Z_{1}}^{Z_{j}}\left[1+M_{G}\left(Z_{j}-x\right)\right] \mathrm{d} G(x)=c_{1}$

Denote the left-hand side of equation (6) by $Q_{1}\left(Z_{1}\right)$,

$$
\begin{gathered}
Q_{1}(0)=0 \\
Q_{1}\left(Z_{2}\right)=\sum_{j=2}^{K}\left(c_{j}-c_{j-1}\right) \int_{Z_{j}-Z_{2}}^{Z_{j}}\left[1+M_{G}\left(Z_{j}-x\right)\right] \mathrm{d} G(x) .
\end{gathered}
$$

Thus, if $Q_{1}\left(Z_{2}\right)>c_{1}$, then there exists a unique and finite $Z^{*}{ }_{1}\left(0<Z_{1}^{*}<Z_{2}\right)$ which satisfies (6). When $K=2,(6)$ is:

$\int_{Z_{2}-Z_{1}}^{Z_{2}}\left[1+M_{G}\left(Z_{2}-x\right)\right] \mathrm{d} G(x)=\frac{c_{1}}{c_{2}-c_{1}}$

whose left-hand side increases strictly with $Z_{1}$ from 0 to $M_{G}\left(Z_{2}\right)$. Thus, if $M_{G}\left(Z_{2}-x\right)>c_{1} /\left(c_{2}-\right.$ $c_{1}$ ), then there exists a unique and finite $Z_{1}^{*}$ which satisfies (7). It can be easily shown that $Z_{1}^{*}$ in (6) decreases with $K$ to $Z_{1}$ in (7).

When $c_{K}>c_{K-1}=c_{K-2}=\cdots=c_{2}>c_{1}$, the expected cost rate in (5) is:

$$
T C_{1}\left(Z_{1}\right)=\frac{\begin{array}{r}
c_{1}-\left(c_{K}-c_{2}\right)\left[\bar{G}\left(Z_{K}\right)-\int_{0}^{Z_{1}} \bar{G}\left(Z_{K}-x\right) \mathrm{d} M_{G}(x)\right] \\
+\left(c_{2}-c_{1}\right)\left[\bar{G}\left(Z_{2}\right)-\int_{0}^{Z_{1}} \bar{G}\left(Z_{2}-x\right) d M_{G}(x)\right]
\end{array}}{1+M_{G}\left(Z_{1}\right)}
$$

and (6) is rewritten as;

$$
\begin{aligned}
& \left(c_{K}-c_{2}\right) \int_{Z_{K}-Z_{1}}^{Z_{K}}\left[1+M_{G}\left(Z_{K}-x\right)\right] \mathrm{d} G(x)+\left(c_{2}-c_{1}\right) \int_{Z_{2}-Z_{1}}^{Z_{2}}\left[1+M_{G}\left(Z_{2}-x\right)\right] d G(x) \\
& =c_{1}
\end{aligned}
$$

whose left-hand side $Q_{1}\left(Z_{1}\right)$ increases strictly with $Z_{1}$ from 0 to

$$
\begin{gathered}
Q_{1}\left(Z_{2}\right)=\left(c_{K}-c_{2}\right) \int_{Z_{K}-Z_{2}}^{Z_{K}}\left[1+M_{G}\left(Z_{K}-x\right)\right] \mathrm{d} G(x) \\
+\left(c_{2}-c_{1}\right) \int_{0}^{Z_{2}}\left[1+M_{G}\left(Z_{2}-x\right)\right] d G(x) \\
=\left(c_{K}-c_{2}\right) \int_{Z_{K}-Z_{2}}^{Z_{K}}\left[1+M_{G}\left(Z_{K}-x\right)\right] \mathrm{d} G(x)+\left(c_{2}-c_{1}\right) M_{G}\left(Z_{2}\right) .
\end{gathered}
$$


Thus, if $Q_{1}\left(Z_{2}\right)>c_{1}$, then there exists a unique and finite $Z_{1}^{*}\left(0<Z_{1}^{*}<Z_{2}\right)$ which satisfies (9). It can be easily shown that $Z_{1}{ }_{1}$ in (6) is smaller than $Z_{1}^{*}$ in (9). When $G(x)=1-\exp (-\mu x)$, the expected cost rate (5) is rewritten as;

$T C_{1}\left(Z_{1}\right)=\frac{c_{1}+\sum_{j=2}^{K}\left(c_{j}-c_{j-1}\right) \mathrm{e}^{-\mu\left(Z_{j}-Z_{1}\right)}}{1+\mu Z_{1}}$

and (6) is rewritten as;

$\sum_{j=2}^{K}\left(c_{j}-c_{j-1}\right) \mu Z_{1} \mathrm{e}^{-\mu\left(Z_{j}-Z_{1}\right)}=c_{1}$

Letting denote the left-hand side of (11) by $Q_{1}\left(Z_{1}\right), Q_{1}\left(Z_{1}\right)$ increases strictly with $Z_{1}$ from 0 to

$Q_{1}\left(Z_{2}\right)=\sum_{j=2}^{K}\left(c_{j}-c_{j-1}\right) \mu Z_{2} \mathrm{e}^{-\mu\left(Z_{j}-Z_{2}\right)}$

Thus, if $Q_{1}\left(Z_{2}\right)>c_{1}$, then there exists a unique and finite $Z_{1}{ }_{1}\left(0<Z_{1}{ }_{1}<Z_{2}\right)$ which satisfies (11).

In particular, when $K=2,(11)$ is:

$\mu Z_{1} \mathrm{e}^{-\mu\left(Z_{2}-Z_{1}\right)}=\frac{c_{1}}{c_{2}-c_{1}}$

If $\mu Z_{2}>c_{1} /\left(c_{2}-c_{1}\right)$, then there exists a unique and finite $Z_{1}^{*}\left(0<Z_{1}^{*}<Z_{2}\right)$ which satisfies (13).

\section{Model 2}

We consider the following maintenance policy and assumptions (1) and (3) in Section 2.

2') The infrastructure damage levels are denoted as discrete levels $Z_{i}(i=1,2, \cdots, K-1, K)$. The $\mathrm{CM}$ is undergone at time $j T$ when the cumulative damage surpasses a threshold level $Z_{K}$ at time $j T$. When the cumulative damage is between $Z_{1}\left(<Z_{K}\right)$ and $Z_{K}$, the PM is undergone at time $(j+1) T$.

The probability that $\mathrm{CM}$ is undergone at time $(j+2) T$ is:

$\sum_{j=0}^{\infty} \sum_{i=1}^{K-1} \int_{0}^{Z_{1}}\left[\int_{Z_{i}-x}^{Z_{i+1}-x} \bar{G}\left(Z_{K}-x-y\right) \mathrm{d} G(y)\right] \mathrm{d} G^{(j)}(x)$

the probabilities that $\mathrm{PM}$ is undergone at time $(j+2) T$ is:

$\sum_{i=1}^{K-1} \sum_{j=0}^{\infty} \int_{0}^{Z_{1}}\left[\int_{Z_{i}-x}^{Z_{i+1}-x} G\left(Z_{i+1}-x-y\right) \mathrm{d} G(y)\right] \mathrm{d} G^{(j)}(x)$
$+\sum_{i=1}^{K-1} \sum_{j=0}^{\infty} \int_{0}^{Z_{1}}\left\{\sum_{n=i+1}^{K-1} \int_{Z_{i}-x}^{Z_{i+1}-x}\left[G\left(Z_{n+1}-x-y\right)-G\left(Z_{n}-x-y\right)\right] \mathrm{d} G(y)\right\} \mathrm{d} G^{(j)}(x)$

where, $\phi^{(j)}(x)(j=1,2, \cdots)$ denotes the $j$-fold Stieltjes convolution of distribution $\phi(x), \phi^{(0)}(x) \equiv$ 1 , and $(1)+(14)+(15)=1$. The mean time to maintenance is: 
$\sum_{j=0}^{\infty}(j+1) T \int_{0}^{Z_{1}} \bar{G}\left(Z_{K}-x\right) \mathrm{d} G^{(j)}(x)$

$+\sum_{i=1}^{K-1} \sum_{j=0}^{\infty}(j+2) T \int_{0}^{Z_{1}}\left[\int_{Z_{i}-x}^{Z_{i+1}-x} \bar{G}\left(Z_{K}-x-y\right) \mathrm{d} G(y)\right] \mathrm{d} G^{(j)}(x)$

$+\sum_{i=1}^{K-1} \sum_{j=0}^{\infty}(j+2) \mathrm{T} \int_{0}^{Z_{1}}\left[\int_{Z_{i}-x}^{Z_{i+1}-x} G\left(Z_{i+1}-x-y\right) \mathrm{d} G(y)\right] \mathrm{d} G^{(j)}(x)$

$+\sum_{i=1}^{K-1} \sum_{j=0}^{\infty}(j+2) T \int_{0}^{Z_{1}}\left\{\sum_{n=i+1}^{K-1} \int_{Z_{i}-x}^{Z_{i+1}-x}\left[G\left(Z_{n+1}-x-y\right)-G\left(Z_{n}-x-y\right)\right] \mathrm{d} G(y)\right\} \mathrm{d} G^{(j)}(x)$

$=T\left[1+G\left(Z_{K}\right)+\int_{0}^{Z_{1}} G\left(Z_{K}-x\right) \mathrm{d} M_{G}(x)\right]$

where, $M_{G}(x) \equiv \sum_{j=1}^{\infty} G^{(j)}(x)$. The total expected cost until maintenance is:

$c_{K} \sum_{j=0}^{\infty} \int_{0}^{Z_{1}} \bar{G}\left(Z_{K}-x\right) \mathrm{d} G^{(j)}(x)+c_{K} \sum_{i=1}^{K-1} \sum_{j=0}^{\infty} \int_{0}^{Z_{1}}\left[\int_{Z_{i}-x}^{Z_{i+1}-x} \bar{G}\left(Z_{K}-x-y\right) \mathrm{d} G(y)\right] \mathrm{d} G^{(j)}(x)$

$+\sum_{i=1}^{K-1} \sum_{j=0}^{\infty} c_{i} \int_{0}^{Z_{1}}\left[\int_{Z_{i}-x}^{Z_{i+1}-x} G\left(Z_{i+1}-x-y\right) \mathrm{d} G(y)\right] \mathrm{d} G^{(j)}(x)$

$+\sum_{i=1}^{K-1} \sum_{j=0}^{\infty} \int_{0}^{Z_{1}}\left\{\sum_{n=i+1}^{K-1} c_{n} \int_{Z_{i}-x}^{Z_{i+1}-x}\left[G\left(Z_{n+1}-x-y\right)-G\left(Z_{n}-x-y\right)\right] \mathrm{d} G(y)\right\} \mathrm{d} G^{(j)}(x)$

$=c_{K}-\sum_{i=1}^{K-1}\left(c_{K}-c_{i}\right) \sum_{j=0}^{\infty} \int_{0}^{Z_{1}}\left[\int_{Z_{i}-x}^{Z_{i+1}-x} G\left(Z_{i+1}-x-y\right) \mathrm{d} G(y)\right] \mathrm{d} G^{(j)}(x)$

$-\sum_{i=1}^{K-1} \sum_{j=0}^{\infty} \int_{0}^{Z_{1}}\left\{\sum_{n=i+1}^{K-1}\left(c_{K}-c_{n}\right) \int_{Z_{i}-x}^{Z_{i+1}-x}\left[G\left(Z_{n+1}-x-y\right)-G\left(Z_{n}-x-y\right)\right] \mathrm{d} G(y)\right\} \mathrm{d} G^{(j)}(x)$

$=c_{K}-\sum_{j=2}^{K}\left(c_{j}-c_{j-1}\right)\left\{\int_{Z_{1}}^{Z_{\mathrm{j}}} G\left(Z_{j}-x\right) \mathrm{d} G(x)\right.$

$\left.+\int_{0}^{Z_{1}}\left[\int_{Z_{1}-x}^{Z_{j}-x} G\left(Z_{j}-x-y\right) \mathrm{d} G(y)\right] \mathrm{d} M_{G}(x)\right\}$

Thus, the expected cost rate is from (16) and (17), 
$c_{K}-\sum_{j=2}^{K}\left(c_{j}-c_{j-1}\right)\left\{\int_{Z_{1}}^{Z_{j}} G\left(Z_{j}-x\right) \mathrm{d} G(x)\right.$
$T C_{2}\left(Z_{1}\right)=\frac{\left.+\int_{0}^{Z_{1}}\left[\int_{Z_{1}-x}^{Z_{j}-x} G\left(Z_{j}-x-y\right) \mathrm{d} G(y)\right] \mathrm{d} M_{G}(x)\right\}}{1+G\left(Z_{K}\right)+\int_{0}^{Z_{1}} G\left(Z_{K}-x\right) \mathrm{d} M_{G}(x)}$

Because it is hard to consider optimal policies analytically, it is assumed that $G(x)=1-\exp (-\mu x)$. Then, (18) is rewritten as;

$T C_{2}\left(Z_{1}\right)=\frac{c_{1}+\sum_{j=2}^{K}\left(c_{j}-c_{j-1}\right)\left[1+\mu\left(Z_{j}-Z_{1}\right)\right] e^{-\mu\left(Z_{j}-Z_{1}\right)}}{2+\mu Z_{1}-\mathrm{e}^{-\mu\left(Z_{K}-Z_{1}\right)}}$

We seek an optimal $Z_{1}^{*}$ which minimizes $C_{2}\left(Z_{1}\right)$. Differentiating $T C_{2}\left(Z_{1}\right)$ with respect to $Z_{1}$ and setting it equal to zero;

$\sum_{j=2}^{K}\left(c_{j}-c_{j-1}\right) \mathrm{e}^{-\mu\left(Z_{j}-Z_{1}\right)}\left[\left(1+\mu Z_{1}\right) \frac{\mu\left(Z_{j}-Z_{1}\right)}{1-e^{-\mu\left(Z_{K}-Z_{1}\right)}}-1\right]=c_{1}$

When $\mu\left(Z_{j}-Z_{1}\right)=1-\exp \left[\mu\left(Z_{j}-Z_{1}\right)\right]$, the left-hand side of (20) agrees with that of (11). In particular, When $K=2$, (20) is:

$e^{-\mu\left(Z_{2}-Z_{1}\right)}\left[\left(1+\mu Z_{1}\right) \frac{\mu\left(Z_{2}-Z_{1}\right)}{1-e^{-\mu\left(Z_{2}-Z_{1}\right)}}-1\right]=\frac{c_{1}}{c_{2}-c_{1}}$

(21) is equivalent to eq. (13) of (see Ito and Nakagawa, 2018). Comparing (21) with (13), $Z_{1}^{*}$ in (21) is smaller than that in (13), because $\mu\left(Z_{2}-Z_{1}\right) \geq 1-\exp \left[-\mu\left(Z_{2}-Z_{1}\right)\right]$.

In general, we cannot prove that the monotonous property of the left-hand side of (20) for $Z_{1}$. So that, we consider the following three approximate $\tilde{Z}_{1}$ cases as follows:

\subsection{Case I:}

$\sum_{j=2}^{K}\left(c_{j}-c_{j-1}\right) \mathrm{e}^{-\mu\left(Z_{j}-Z_{1}\right)}\left[\left(1+\mu Z_{1}\right) \frac{Z_{j}-Z_{1}}{Z_{K}-Z_{1}}-1\right]=c_{1}$

Letting denote the left-hand sides of (20) and (22) by $Q_{2}\left(Z_{1}\right)$ and $Q_{21}\left(Z_{1}\right)$, respectively. $Q_{2}\left(Z_{1}\right)>$ $Q_{21}\left(Z_{1}\right)$ because $\mu\left(Z_{K}-Z_{1}\right)>1-\exp \left[-\mu\left(Z_{K}-Z_{1}\right)\right] . Z_{1}^{*}<\tilde{Z}_{1}$ where $Z_{1}^{*}$ satisfies (20) and $\tilde{Z}_{1}$ satisfies (22). When $K=2, \tilde{Z}_{1}$ is equal to $Z_{1}^{*}$ which satisfies (13).

\subsection{Case II:}

$\sum_{j=2}^{K}\left(c_{j}-c_{j-1}\right) \mathrm{e}^{-\mu\left(Z_{j}-Z_{1}\right)}\left[\left(1+\mu Z_{1}\right) \frac{\mu\left(Z_{j}-Z_{1}\right)}{1-e^{-\mu\left(Z_{K}-Z_{1}\right)}}-1\right]=c_{1}$

Letting denote the left-hand sides of (23) by $Q_{22}\left(Z_{1}\right), Q_{2}\left(Z_{1}\right)<Q_{22}\left(Z_{1}\right)$ because $1-\exp \left[-\mu\left(Z_{K}-Z_{1}\right)\right]$ $>1-\exp \left[-\mu\left(Z_{j}-Z_{1}\right)\right] . Z_{1}^{*}>\tilde{Z}_{1}$ where $Z_{1}^{*}$ satisfies (20) and $\tilde{Z}_{1}$ satisfies (23). 


\subsection{Case III:}

$$
\left[\left(1+\mu Z_{1}\right) \frac{\mu\left(Z_{j}-Z_{1}\right)}{1-e^{-\mu\left(Z_{K}-Z_{1}\right)}}-1\right] \sum_{j=2}^{K}\left(c_{j}-c_{j-1}\right) \mathrm{e}^{-\mu\left(Z_{j}-Z_{1}\right)}=c_{1}
$$

Letting denote the left-hand sides of (23) by $Q_{23}\left(Z_{1}\right), Q_{2}\left(Z_{1}\right)<Q_{23}\left(Z_{1}\right)$ because $\mu\left(Z_{K}-Z_{1}\right)>\mu\left(Z_{j}-\right.$ $\left.Z_{1}\right) . Z_{1}^{*}>\tilde{Z}_{1}$ where $Z_{1}^{*}$ satisfies (20) and $\tilde{Z}_{1}$ satisfies (24).

Because

$$
\frac{\mu\left(Z_{j}-Z_{1}\right)}{1-e^{-\mu\left(Z_{j}-Z_{1}\right)}} \geq\left[\frac{\mu\left(Z_{K}-Z_{1}\right)}{1-e^{-\mu\left(Z_{K}-Z_{1}\right)}}\right]
$$

$\tilde{Z}_{1}$ which satisfies (23) is greater than $\tilde{Z}_{1}$ which satisfies (24).

\section{Model 3}

We consider the following maintenance policy and assumptions 1) and 3) in Section 2.

2 ''). The infrastructure damage levels are denoted as discrete levels $Z_{i}(i=1,2, \cdots, L-1, L, \cdots, K$

$-1, K)$. The $\mathrm{CM}$ is undergone at time $j T$ when the cumulative damage surpasses a threshold level $Z_{K}$ at time $j T$. When the cumulative damage is between $Z_{L}\left(<Z_{K}\right)$ and $Z_{K}$ the PM is undergone at time $(j+1) T$ when the cumulative damage is between $Z_{1}\left(<Z_{L}\right)$ and $Z_{L}$, and is undergone at time $j T$.

The probability that $\mathrm{CM}$ is undergone at time $(j+2) T$ is:

$\sum_{i=1}^{L-1} \sum_{j=0}^{\infty} \int_{0}^{Z_{1}}\left[\int_{Z_{i}-x}^{Z_{i+1}-x} \bar{G}\left(Z_{K}-x-y\right) \mathrm{d} G(y)\right] \mathrm{d} G^{(j)}(x)$

The probabilities that PM is undergone at time $(j+2) T$ is:

$$
\begin{aligned}
& \sum_{i=1}^{L-1} \sum_{j=0}^{\infty} \int_{0}^{Z_{1}}\left[\int_{Z_{i}-x}^{Z_{i+1}-x} G\left(Z_{i+1}-x-y\right) \mathrm{d} G(y)\right] \mathrm{d} G^{(j)}(x) \\
& +\sum_{i=1}^{L-1} \sum_{j=0}^{\infty} \int_{0}^{Z_{1}}\left\{\sum_{n=i+1}^{K-1} \int_{Z_{i}-x}^{Z_{i+1}-x}\left[G\left(Z_{n+1}-x-y\right)-G\left(Z_{n}-x-y\right)\right] \mathrm{d} G(y)\right\} \mathrm{d} G^{(j)}(x) \\
& +\sum_{i=L}^{K-1} \sum_{j=0}^{\infty} \int_{0}^{Z_{1}}\left[G\left(Z_{i+1}-x\right)-G\left(Z_{i}-x\right)\right] \mathrm{d} G^{(j)}(x)
\end{aligned}
$$

where, $(1)+(25)+(26)=1$. The mean time to maintenance is:

$$
\begin{aligned}
& \sum_{j=0}^{\infty}(j+1) T \int_{0}^{Z_{1}} \bar{G}\left(Z_{K}-x\right) \mathrm{d} G^{(j)}(x) \\
& +\sum_{i=1}^{L-1} \sum_{j=0}^{\infty}(j+2) T \int_{0}^{Z_{1}}\left[\int_{Z_{i}-x}^{Z_{i+1}-x} \bar{G}\left(Z_{K}-x-y\right) \mathrm{d} G(y)\right] \mathrm{d} G^{(j)}(x)
\end{aligned}
$$


$+\sum_{i=1}^{L-1} \sum_{j=0}^{\infty}(j+2) T \int_{0}^{Z_{1}}\left[\int_{Z_{i}-x}^{Z_{i+1}-x} G\left(Z_{i+1}-x-y\right) \mathrm{d} G(y)\right] \mathrm{d} G^{(j)}(x)$

$+\sum_{i=1}^{L-1} \sum_{j=0}^{\infty}(j+2) T \int_{0}^{Z_{1}}\left\{\sum_{n=i+1}^{K-1} \int_{Z_{i}-x}^{Z_{i+1}-x}\left[G\left(Z_{n+1}-x-y\right)-G\left(Z_{n}-x-y\right)\right] \mathrm{d} G(y)\right\} \mathrm{d} G^{(j)}(x)$

$+\sum_{i=L}^{K-1} \sum_{j=0}^{\infty}(j+1) T \int_{0}^{Z_{1}}\left[G\left(Z_{i+1}-x\right)-G\left(Z_{i}-x\right)\right] \mathrm{d} G^{(j)}(x)$

$=T\left[1+G\left(Z_{L}\right)+\int_{0}^{Z_{1}} G\left(Z_{L}-x\right) \mathrm{d} M_{G}(x)\right]$

The total expected cost until maintenance is:

$$
\begin{aligned}
& c_{K} \sum_{j=0}^{\infty} \int_{0}^{Z_{1}} \bar{G}\left(Z_{K}-x\right) \mathrm{d} G^{(j)}(x)+c_{K} \sum_{i=1}^{L-1} \sum_{j=0}^{\infty} \int_{0}^{Z_{1}}\left[\int_{Z_{i}-x}^{Z_{i+1}-x} \bar{G}\left(Z_{K}-x-y\right) \mathrm{d} G(y)\right] \mathrm{d} G^{(j)}(x) \\
& +\sum_{i=1}^{L-1} \sum_{j=0}^{\infty} c_{i} \int_{0}^{Z_{1}}\left[\int_{Z_{i}-x}^{Z_{i+1}-x} G\left(Z_{i+1}-x-y\right) \mathrm{d} G(y)\right] \mathrm{d} G^{(j)}(x) \\
& +\sum_{i=L}^{K-1} c_{i} \sum_{j=0}^{\infty} \int_{0}^{Z_{1}}\left[G\left(Z_{i+1}-x\right)-G\left(Z_{i}-x\right)\right] \mathrm{d} G^{(j)}(x) \\
& +\sum_{i=1}^{L-1} \sum_{j=0}^{\infty} \int_{0}^{Z_{1}}\left\{\sum_{n=i+1}^{K-1} c_{n} \int_{Z_{i}-x}^{Z_{i+1}-x}\left[G\left(Z_{n+1}-x-y\right)-G\left(Z_{n}-x-y\right)\right] \mathrm{d} G(y)\right\} \mathrm{d} G^{(j)}(x) \\
& =c_{K}-\sum_{i=\mathrm{L}}^{K-1} \sum_{j=0}^{\infty}\left(c_{K}-c_{i}\right) \int_{0}^{Z_{1}}\left[G\left(Z_{i+1}-x\right)-G\left(Z_{i}-x\right)\right] \mathrm{d} G^{(j)}(x) \\
& -\sum_{i=1}^{L-1} \sum_{j=0}^{\infty} \int_{0}^{Z_{1}}\left\{\sum_{n=i+1}^{K-1}\left(c_{K}-c_{n}\right) \int_{Z_{i}-x}^{Z_{i+1}-x}\left[G\left(Z_{n+1}-x-y\right)-G\left(Z_{n}-x-y\right)\right] \mathrm{d} G(y)\right\} \mathrm{d} G^{(j)}(x) \\
& -\sum_{i=1}^{L-1} \sum_{j=0}^{\infty}\left(c_{K}-c_{i}\right) \int_{0}^{Z_{1}}\left[\int_{Z_{i}-x}^{Z_{i+1}-x} G\left(Z_{i+1}-x-y\right) \mathrm{d} G(y)\right] \mathrm{d} G^{(j)}(x) \\
& =c_{K}-\sum_{i=L+1}^{K}\left(c_{i}-c_{i+1}\right)\left\{G\left(Z_{i}\right)-G\left(Z_{L}\right)+\int_{0}^{Z_{1}}\left[G\left(Z_{i}-x\right)-G\left(Z_{L}-x\right)\right] \mathrm{d} M_{G}(x)\right\} \\
& -\sum_{i=2}^{L} \sum_{j=i}^{K}\left(c_{j}-c_{j-1}\right)\left\{\int_{Z_{i-1}}^{Z_{i}} G\left(Z_{j}-x\right) \mathrm{d} G(x)\right.
\end{aligned}
$$


$\left.+\int_{0}^{Z_{1}}\left[\int_{Z_{i-1}-x}^{Z_{i}-x} G\left(Z_{j}-x-y\right) \mathrm{d} G(y)\right] \mathrm{d} M_{G}(x)\right\}$

Thus, the expected cost rate is from (27) and (28),

$$
\begin{array}{r}
c_{K}-\sum_{i=\mathrm{L}+1}^{K}\left(c_{i}-c_{i-1}\right)\left\{G\left(Z_{i}\right)-G\left(Z_{L}\right)\right. \\
\left.+\int_{0}^{Z_{1}}\left[G\left(Z_{i}-x\right)-G\left(Z_{L}-x\right)\right] \mathrm{d} M_{G}(x)\right\} \\
-\sum_{i=2}^{L} \sum_{j=i}^{K}\left(c_{j}-c_{j-1}\right)\left\{\int_{Z_{i-1}}^{Z_{i}} G\left(Z_{j}-x\right) \mathrm{d} G(x)\right. \\
T C_{3}\left(Z_{1}\right)=\frac{\left.+\int_{0}^{Z_{1}}\left[\int_{Z_{i-1}-x}^{Z_{i}-x} G\left(Z_{j}-x-y\right) \mathrm{d} G(y)\right] \mathrm{d} M_{G}(x)\right\}}{1+G\left(Z_{L}\right)+\int_{0}^{Z_{1}} G\left(Z_{L}-x\right) \mathrm{d} M_{G}(x)}
\end{array}
$$

Because it is difficult to discuss optimal policies analytically, it is assumed that $G(x)=1-$ $\exp (-\mu x)$. Then, (29) is rewritten as;

$T C_{3}\left(Z_{1}\right)=\frac{+\sum_{i=2}^{L} \sum_{j=i}^{K}\left(c_{j}-c_{j-1}\right) \mu\left(Z_{i}-Z_{i-1}\right) \mathrm{e}^{-\mu\left(Z_{j}-Z_{1}\right)}}{2+\mu Z_{1}-\mathrm{e}^{-\mu\left(Z_{L}-Z_{1}\right)}}$

We seek an optimal $Z_{1}^{*}$ which minimizes $C_{3}\left(Z_{1}\right)$. Differentiating (30) with respect to $Z_{1}$ and setting it equal to zero,

$$
\begin{aligned}
& \sum_{i=2}^{L}\left(c_{i}-c_{i-1}\right) \mathrm{e}^{-\mu\left(Z_{i}-Z_{1}\right)}\left[\frac{\left(1+\mu Z_{1}\right) \mu\left(Z_{i}-Z_{1}\right)}{1-e^{-\mu\left(Z_{L}-Z_{1}\right)}}-1\right] \\
& -\left[\frac{\left(1+\mu Z_{1}\right) \mu\left(Z_{L}-Z_{1}\right)}{1-e^{-\mu\left(Z_{L}-Z_{1}\right)}}-1\right] \sum_{i=L+1}^{K}\left(c_{i}-c_{i-1}\right) \mathrm{e}^{-\mu\left(Z_{i}-Z_{1}\right)}=c_{1}
\end{aligned}
$$

When $L=K$, (31) is equivalent to (20) where $\sum_{i=K+1}^{K} \equiv 0$.

\section{Extended Models}

We expand Model 1, 2, and 3 to models which consider the natural disaster.

\subsection{Model 4}

We make assumptions 1), 2), and 3) of Section 2 and add the following assumption:

4). The social infrastructure is damaged by the natural disaster and the severe weather which occur independently of normal degradations, and the disaster recovery cost is much higher than the CM cost. The occurrence time of the natural disaster has a general distribution $F(t)$, and the average disaster recovery cost is $c_{D}\left(>c_{K}\right)$.

The probability that $\mathrm{CM}$ is undergone at time $(j+1) T$ is:

$$
\sum_{j=0}^{\infty} \bar{F}((j+1) T) \int_{0}^{Z_{1}} \bar{G}\left(Z_{K}-x\right) \mathrm{d} G^{(j)}(x)
$$


the probability that $\mathrm{PM}$ is undergone at time $(j+1) T$ is:

$\sum_{i=1}^{K-1} \sum_{j=0}^{\infty} \bar{F}((j+1) T) \int_{0}^{Z_{1}}\left[G\left(Z_{i+1}-x\right)-G\left(Z_{i}-x\right)\right] \mathrm{d} G^{(j)}(x)$

and the probability that the disaster recovery occurs at time $(j+1) T$ is:

$\sum_{j=0}^{\infty} F((j+1) T) \int_{0}^{Z_{1}} \bar{G}\left(Z_{1}-x\right) \mathrm{d} G^{(j)}(x)$

The mean time to maintenance is:

$$
\begin{aligned}
& \sum_{j=0}^{\infty}(j+1) T \bar{F}((j+1) T) \int_{0}^{Z_{1}} \bar{G}\left(Z_{K}-x\right) \mathrm{d} G^{(j)}(x) \\
& +\sum_{i=1}^{K-1} \sum_{j=0}^{\infty}(j+1) T \bar{F}((j+1) T) \int_{0}^{Z_{1}}\left[G\left(Z_{i+1}-x\right)-G\left(Z_{i}-x\right)\right] \mathrm{d} G^{(j)}(x) \\
& +\sum_{j=0}^{\infty} \int_{0}^{(j+1) T} t \mathrm{~d} F(t) \int_{0}^{Z_{1}} \bar{G}\left(Z_{1}-x\right) \mathrm{d} G^{(j)}(x)=\sum_{j=0}^{\infty} G^{(j)}\left(Z_{1}\right) \int_{j T}^{(j+1) T} \bar{F}(t) \mathrm{d} t
\end{aligned}
$$

and the total expected cost until maintenance is:

$$
\begin{aligned}
& c_{K} \sum_{j=0}^{\infty} \bar{F}((j+1) T) \int_{0}^{Z_{1}} \bar{G}\left(Z_{K}-x\right) \mathrm{d} G^{(j)}(x) \\
& +\sum_{i=1}^{K-1} c_{i} \sum_{j=0}^{\infty} \bar{F}((j+1) T) \int_{0}^{Z_{1}}\left[G\left(Z_{i+1}-x\right)-G\left(Z_{i}-x\right)\right] \mathrm{d} G^{(j)}(x) \\
& +c_{D} \sum_{j=0}^{\infty} F((j+1) T) \int_{0}^{Z_{1}} \bar{G}\left(Z_{1}-x\right) \mathrm{d} G^{(j)}(x) \\
& =c_{K}-\sum_{i=1}^{K-1}\left(c_{K}-c_{i}\right) \sum_{j=0}^{\infty} \bar{F}((j+1) T) \int_{0}^{Z_{1}}\left[G\left(Z_{i+1}-x\right)-G\left(Z_{i}-x\right)\right] \mathrm{d} G^{(j)}(x) \\
& +\left(c_{D}-c_{K}\right) \sum_{j=0}^{\infty} F((j+1) T)\left[G^{(j)}\left(Z_{1}\right)-G^{(j+1)}\left(Z_{1}\right)\right] \\
& =c_{K}-\sum_{i=2}^{K}\left(c_{i}-c_{i-1}\right) \sum_{j=0}^{\infty} \bar{F}((j+1) T) \int_{0}^{Z_{1}} G\left(Z_{i}-x\right) \mathrm{d} G^{(j)}(x)+\left(c_{K}-c_{1}\right) \sum_{j=1}^{\infty} \bar{F}(j T) G^{(j)}\left(Z_{1}\right) \\
& +\left(c_{D}-c_{K}\right) \sum_{j=0}^{\infty} F((j+1) T)\left[G^{(j)}\left(Z_{1}\right)-G^{(j+1)}\left(Z_{1}\right)\right]
\end{aligned}
$$


Thus, the expected cost rate is, from (35) and (36),

$$
C_{4}\left(Z_{1}\right)=\frac{\begin{array}{c}
c_{K}-\sum_{i=2}^{K}\left(c_{i}-c_{i-1}\right) \sum_{j=0}^{\infty} \bar{F}((j+1) T) \int_{0}^{Z_{1}} G\left(Z_{i}-x\right) \mathrm{d} G^{(j)}(x) \\
+\left(c_{K}-c_{1}\right) \sum_{j=1}^{\infty} \bar{F}(j T) G^{(j)}\left(Z_{1}\right) \\
+\left(c_{D}-c_{K}\right) \sum_{j=0}^{\infty} F((j+1) T)\left[G^{(j)}\left(Z_{1}\right)-G^{(j+1)}\left(Z_{1}\right)\right]
\end{array}}{\sum_{j=0}^{\infty} G^{(j)}\left(Z_{1}\right) \int_{j T}^{(j+1) T} \bar{F}(t) \mathrm{d} t}
$$

When $F(t)=1-\exp (-\lambda t)$ and $G(x)=1-\exp (-\mu x),(37)$ is:

$\frac{C_{4}\left(Z_{1}\right)}{\lambda}=\frac{c_{K}-\alpha \mathrm{e}^{-(1-\alpha) \mu Z_{1}} \sum_{i=2}^{K}\left(c_{i}-c_{i-1}\right)\left[1-\mathrm{e}^{-\mu\left(Z_{i}-Z_{1}\right)}\right]}{1-\alpha \mathrm{e}^{-(1-\alpha) \mu Z_{1}}}+c_{D}-c_{K}$

where, $\alpha \equiv \exp (-\lambda T)$. We find optimal $Z_{1}^{*}$ to minimize $C_{4}\left(Z_{1}\right)$. Differentiating $C_{4}\left(Z_{1}\right)$ with $Z_{1}$ and putting it equal to zero,

$\frac{\alpha}{1-\alpha}\left[1-\mathrm{e}^{-(1-\alpha) \mu Z_{1}}\right] \sum_{i=2}^{K}\left(c_{i}-c_{i-1}\right) \mathrm{e}^{-\mu\left(Z_{i}-Z_{1}\right)}=c_{1}$

Letting denote the left-hand side of equation (39) by $Q_{4}\left(Z_{1} ; \alpha\right)$,

$$
\begin{gathered}
Q_{4}(0 ; \alpha)=0 \\
Q_{4}\left(Z_{2} ; \alpha\right)=\frac{\alpha}{1-\alpha}\left[1-\mathrm{e}^{-(1-\alpha) \mu Z_{2}}\right]\left[c_{2}-c_{1}-\sum_{i=3}^{K}\left(c_{i}-c_{i-1}\right) \mathrm{e}^{-\mu\left(Z_{i}-Z_{2}\right)}\right] .
\end{gathered}
$$

Thus, if $Q_{4}\left(Z_{2}\right)>c_{1}$, then there exists a unique and finite $Z_{1}{ }_{1}\left(0<Z_{1}{ }_{1}<Z_{2}\right)$ which satisfies (39).

When $K=2$, (12) is equivalent to eq. (22) of (see Ito et al., 2018).

\subsection{Model 5}

We make assumptions 1) and 3) of Section 2, assumption 2') of Section 3, and assumption 4) of Section 5.1. The mean time to maintenance is:

$$
\begin{aligned}
& \sum_{j=0}^{\infty}(j+1) T \bar{F}((j+1) T) \int_{0}^{Z_{1}} \bar{G}\left(Z_{K}-x\right) \mathrm{d} G^{(j)}(x) \\
& +\sum_{j=0}^{\infty}(j+2) T \bar{F}((j+2) T) \sum_{i=1}^{K-1} \int_{0}^{Z_{1}}\left[\int_{Z_{i}-x}^{Z_{i+1}-x} \bar{G}\left(Z_{K}-x-y\right) \mathrm{d} G(y)\right] \mathrm{d} G^{(j)}(x) \\
& +\sum_{j=0}^{\infty}(j+2) T \bar{F}((j+2) T) \sum_{i=1}^{K-1} \int_{0}^{Z_{1}}\left[\int_{Z_{i-1}-x}^{Z_{i}-x} G\left(Z_{i+1}-x-y\right) \mathrm{d} G(y)\right] \mathrm{d} G^{(j)}(x) \\
& +\sum_{j=0}^{\infty}(j+2) T \bar{F}((j+2) T) \sum_{i=1}^{K-1} \int_{0}^{Z_{1}}\left\{\sum _ { n = i + 1 } ^ { K - 1 } \int _ { Z _ { i } - x } ^ { Z _ { i + 1 } - x } \left[G\left(Z_{n+1}-x-y\right)\right.\right.
\end{aligned}
$$


$\left.\left.-G\left(Z_{n}-x-y\right)\right] \mathrm{d} G(y)\right\} \mathrm{d} G^{(j)}(x)$

$+\sum_{j=0}^{\infty} \int_{0}^{(j+1) T} t \mathrm{~d} F(t) \int_{0}^{Z_{1}} \bar{G}\left(Z_{K}-x\right) \mathrm{d} G^{(j)}(x)$

$+\sum_{j=0}^{\infty} \int_{0}^{(j+2) T} t \mathrm{~d} F(t) \sum_{i=1}^{K-1} \int_{0}^{Z_{1}}\left[\int_{Z_{i}-x}^{Z_{i+1}-x} \bar{G}\left(Z_{K}-x-y\right) \mathrm{d} G(y)\right] \mathrm{d} G^{(j)}(x)$

$+\sum_{j=0}^{\infty} \int_{0}^{(j+2) T} t \mathrm{~d} F(t) \sum_{i=1}^{K-1} \int_{0}^{Z_{1}}\left[\int_{Z_{i-1}-x}^{Z_{i}-x} G\left(Z_{i+1}-x-y\right) \mathrm{d} G(y)\right] \mathrm{d} G^{(j)}(x)$

$+\sum_{j=0}^{\infty} \int_{0}^{(j+2) T} t \mathrm{~d} F(t) \sum_{i=1}^{K-1} \int_{0}^{Z_{1}}\left\{\sum_{n=i+1}^{K-1} \int_{Z_{i}-x}^{Z_{i+1}-x}\left[G\left(Z_{n+1}-x-y\right)\right.\right.$

$\left.\left.-G\left(Z_{n}-x-y\right)\right] \mathrm{d} G(y)\right\} \mathrm{d} G^{(j)}(x)$

$=\int_{0}^{T} \bar{F}(t) \mathrm{d} t+\sum_{j=0}^{\infty} \int_{(j+1) T}^{(j+2) T} \bar{F}(t) \mathrm{d} t \int_{0}^{Z_{1}} G\left(Z_{K}-x\right) \mathrm{d} G^{(j)}(x)$

and the total expected cost until maintenance is:

$$
\begin{aligned}
& c_{K} \sum_{j=0}^{\infty} \bar{F}((j+1) T) \int_{0}^{Z_{1}} \bar{G}\left(Z_{K}-x\right) \mathrm{d} G^{(j)}(x) \\
& +c_{K} \sum_{j=0}^{\infty} \bar{F}((j+2) T) \sum_{i=1}^{K-1} \int_{0}^{Z_{1}}\left[\int_{Z_{i}-x}^{Z_{i+1}-x} \bar{G}\left(Z_{K}-x-y\right) \mathrm{d} G(y)\right] \mathrm{d} G^{(j)}(x) \\
& +\sum_{j=0}^{\infty} \bar{F}((j+2) T) \sum_{i=1}^{K-1} c_{i} \int_{0}^{Z_{1}}\left[\int_{Z_{i-1}-x}^{Z_{i}-x} G\left(Z_{i+1}-x-y\right) \mathrm{d} G(y)\right] \mathrm{d} G^{(j)}(x) \\
& +\sum_{j=0}^{\infty} \bar{F}((j+2) T) \sum_{i=1}^{K-1} \int_{0}^{Z_{1}}\left\{\sum _ { n = i + 1 } ^ { K - 1 } c _ { n } \int _ { Z _ { i } - x } ^ { Z _ { i + 1 } - x } \left[G\left(Z_{n+1}-x-y\right)\right.\right. \\
& \left.\left.-G\left(Z_{n}-x-y\right)\right] \mathrm{d} G(y)\right\} \mathrm{d} G^{(j)}(x) \\
& +c_{D}\left(\sum_{j=0}^{\infty} \bar{F}((j+1) T) \int_{0}^{Z_{1}} \bar{G}\left(Z_{K}-x\right) \mathrm{d} G^{(j)}(x)\right. \\
& +\sum_{j=0}^{\infty} \bar{F}((j+2) T) \sum_{i=1}^{K-1} \int_{0}^{Z_{1}}\left[\int_{Z_{i}-x}^{Z_{i+1}-x} \bar{G}\left(Z_{K}-x-y\right) \mathrm{d} G(y)\right] \mathrm{d} G^{(j)}(x)
\end{aligned}
$$




$$
\begin{aligned}
& +\sum_{j=0}^{\infty} \bar{F}((j+2) T) \sum_{i=1}^{K-1} \int_{0}^{Z_{1}}\left[\int_{Z_{i-1}-x}^{Z_{i}-x} G\left(Z_{i+1}-x-y\right) \mathrm{d} G(y)\right] \mathrm{d} G^{(j)}(x) \\
& \left.+\sum_{j=0}^{\infty} \bar{F}((j+2) T) \sum_{i=1}^{K-1} \int_{0}^{Z_{1}}\left\{\sum_{n=i+1}^{K-1} \int_{Z_{i}-x}^{Z_{i+1}-x}\left[G\left(Z_{n+1}-x-y\right)-G\left(Z_{n}-x-y\right)\right] \mathrm{d} G(y)\right\} \mathrm{d} G^{(j)}(x)\right) \\
& =c_{K}+\left(c_{D}-c_{K}\right)\left\{F(T)+\sum_{j=0}^{\infty}[\bar{F}((j+1) T)-\bar{F}((j+2) T)] \int_{0}^{Z_{1}} G\left(Z_{K}-x\right) \mathrm{d} G^{(j)}(x)\right\} \\
& -\sum_{j=0}^{\infty} \bar{F}((j+2) T) \sum_{n=2}^{K}\left(c_{n}-c_{n-1}\right) \int_{0}^{Z_{1}}\left[\int_{Z_{1}-x}^{Z_{n}-x} G\left(Z_{n}-x-y\right) \mathrm{d} G(y)\right] \mathrm{d} G^{(j)}(x)
\end{aligned}
$$

Thus, the expected cost rate is, from (40) and (41),

$$
\begin{gathered}
c_{K}+\left(c_{D}-c_{K}\right)\left\{F(T)+\sum_{j=0}^{\infty}[\bar{F}((j+1) T)-\bar{F}((j+2) T)]\right. \\
\left.\times \int_{0}^{Z_{1}} G\left(Z_{K}-x\right) \mathrm{d} G^{(j)}(x)\right\} \\
-\sum_{j=0}^{\infty} \bar{F}((j+2) T) \sum_{n=2}^{K}\left(c_{n}-c_{n-1}\right) \\
C_{5}\left(Z_{1}\right)=\frac{\times \int_{0}^{Z_{1}}\left[\int_{Z_{1}-x}^{Z_{n}-x} G\left(Z_{n}-x-y\right) \mathrm{d} G(y)\right] \mathrm{d} G^{(j)}(x)}{\int_{0}^{T} \bar{F}(t) d t+\sum_{j=0}^{\infty} \int_{(j+1) T}^{(j+2) T} \bar{F}(t) d t \int_{0}^{Z_{1}} G\left(Z_{K}-x\right) d G^{(j)}(x)} .
\end{gathered}
$$

When $F(t)=1-\exp (-\lambda t)$ and $G(x)=1-\exp (-\mu x)$, (42) is:

$\frac{C_{5}\left(Z_{1}\right)}{\lambda}=\frac{c_{K}-\alpha^{2} \mathrm{e}^{\alpha \mu Z_{1}} \sum_{i=2}^{K}\left(c_{i}-c_{i-1}\right)\left[\mathrm{e}^{-\mu Z_{1}}-\mathrm{e}^{-\mu Z_{i}}-\mu\left(Z_{i}-Z_{1}\right) \mathrm{e}^{-\mu Z_{i}}\right]}{1-\alpha \mathrm{e}^{\alpha \mu Z_{1}}\left[\alpha \mathrm{e}^{-\mu Z_{1}}+(1-\alpha) \mathrm{e}^{-\mu Z_{K}}\right]}+c_{D}-c_{K}$

We find optimal $Z_{1}^{*}$ to minimize $C_{5}\left(Z_{1}\right)$. Differentiating $C_{5}\left(Z_{1}\right)$ with respect to $Z_{1}$ and putting it equal to zero,

$$
\begin{aligned}
& \frac{1}{1-\mathrm{e}^{-\mu\left(Z_{K}-Z_{1}\right)}} \sum_{n=2}^{K}\left(c_{n}-c_{n-1}\right)\left\{\left(1-\alpha \mathrm{e}^{\alpha \mu Z_{1}} \mathrm{e}^{-\mu Z_{K}}\right)\left[1-\mathrm{e}^{-\mu\left(Z_{i}-Z_{1}\right)}\right]\right. \\
& \left.+\frac{\alpha}{1-\alpha}\left[1-\alpha \mathrm{e}^{-(1-\alpha) \mu Z_{1}}\right] \mu\left(Z_{i}-Z_{1}\right) \mathrm{e}^{-\mu\left(Z_{i}-Z_{1}\right)}\right\}=c_{K}
\end{aligned}
$$

When $K=2$, (43) is equivalent to eq. (31) of (Ito et al., 2018).

\subsection{Model 6}

We make assumptions 1) and 3) of Section 2, assumption 2") of Section 4, and assumption 4) of Section 5.1. The mean time to maintenance is:

$$
\sum_{j=0}^{\infty}(j+1) T \bar{F}((j+1) T) \int_{0}^{Z_{1}} \bar{G}\left(Z_{K}-x\right) \mathrm{d} G^{(j)}(x)
$$


$+\sum_{j=0}^{\infty}(j+2) T \bar{F}((j+2) T) \sum_{i=1}^{L-1} \int_{0}^{Z_{1}}\left[\int_{Z_{i}-x}^{Z_{i+1}-x} \bar{G}\left(Z_{K}-x-y\right) \mathrm{d} G(y)\right] \mathrm{d} G^{(j)}(x)$

$+\sum_{j=0}^{\infty}(j+2) T \bar{F}((j+2) T) \sum_{i=1}^{L-1} \int_{0}^{Z_{1}}\left[\int_{Z_{i-1}-x}^{Z_{i}-x} G\left(Z_{i+1}-x-y\right) \mathrm{d} G(y)\right] \mathrm{d} G^{(j)}(x)$

$+\sum_{j=0}^{\infty}(j+2) T \bar{F}((j+2) T) \sum_{i=1}^{L-1} \int_{0}^{Z_{1}}\left\{\sum_{n=i+1}^{K-1} \int_{Z_{i}-x}^{Z_{i+1}-x}\left[G\left(Z_{n+1}-x-y\right)\right.\right.$

$\left.\left.-G\left(Z_{n}-x-y\right)\right] \mathrm{d} G(y)\right\} \mathrm{d} G^{(j)}(x)$

$+\sum_{j=0}^{\infty}(j+1) T \bar{F}((j+1) T) \sum_{i=L}^{K-1} \int_{0}^{Z_{1}}\left[G\left(Z_{i+1}-x\right)-G\left(Z_{i}-x\right)\right] \mathrm{d} G^{(j)}(x)$

$+\sum_{j=0}^{\infty} \int_{0}^{(j+1) T} t \mathrm{~d} F(t) \int_{0}^{Z_{1}} \bar{G}\left(Z_{K}-x\right) \mathrm{d} G^{(j)}(x)$

$+\sum_{j=0}^{\infty} \int_{0}^{(j+2) T} t \mathrm{~d} F(t) \sum_{i=1}^{L-1} \int_{0}^{Z_{1}}\left[\int_{Z_{i}-x}^{Z_{i+1}-x} \bar{G}\left(Z_{K}-x-y\right) \mathrm{d} G(y)\right] \mathrm{d} G^{(j)}(x)$

$+\sum_{j=0}^{\infty} \int_{0}^{(j+2) T} t \mathrm{~d} F(t) \sum_{i=1}^{L-1} \int_{0}^{Z_{1}}\left[\int_{Z_{i-1}-x}^{Z_{i}-x} G\left(Z_{i+1}-x-y\right) \mathrm{d} G(y)\right] \mathrm{d} G^{(j)}(x)$

$+\sum_{j=0}^{\infty} \int_{0}^{(j+2) T} t \mathrm{~d} F(t) \sum_{i=1}^{L-1} \int_{0}^{Z_{1}}\left\{\sum_{n=i+1}^{K-1} \int_{Z_{i}-x}^{Z_{i+1}-x}\left[G\left(Z_{n+1}-x-y\right)\right.\right.$

$\left.\left.-G\left(Z_{n}-x-y\right)\right] \mathrm{d} G(y)\right\} \mathrm{d} G^{(j)}(x)$

$+\sum_{j=0}^{\infty} \int_{0}^{(j+1) T} t \mathrm{~d} F(t) \sum_{i=L}^{K-1} \int_{0}^{Z_{1}}\left[G\left(Z_{i+1}-x\right)-G\left(Z_{i}-x\right)\right] \mathrm{d} G^{(j)}(x)$

$=\int_{0}^{T} \bar{F}(t) \mathrm{d} t+\sum_{j=0}^{\infty} \int_{(j+1) T}^{(j+2) T} \bar{F}(t) \mathrm{d} t \int_{0}^{Z_{1}} G\left(Z_{L}-x\right) \mathrm{d} G^{(j)}(x)$

and the total expected cost until maintenance is:

$$
\begin{aligned}
& c_{K} \sum_{j=0}^{\infty} \bar{F}((j+1) T) \int_{0}^{Z_{1}} \bar{G}\left(Z_{K}-x\right) \mathrm{d} G^{(j)}(x) \\
& +c_{K} \sum_{j=0}^{\infty} \bar{F}((j+2) T) \sum_{i=1}^{L-1} \int_{0}^{Z_{1}}\left[\int_{Z_{i}-x}^{Z_{i+1}-x} \bar{G}\left(Z_{K}-x-y\right) \mathrm{d} G(y)\right] \mathrm{d} G^{(j)}(x)
\end{aligned}
$$


$+\sum_{j=0}^{\infty} \bar{F}((j+2) T) \sum_{i=1}^{L-1} c_{i} \int_{0}^{Z_{1}}\left[\int_{Z_{i-1}-x}^{Z_{i}-x} G\left(Z_{i+1}-x-y\right) \mathrm{d} G(y)\right] \mathrm{d} G^{(j)}(x)$

$+\sum_{j=0}^{\infty} \bar{F}((j+2) T) \sum_{i=1}^{L-1} \int_{0}^{Z_{1}}\left\{\sum_{n=i+1}^{K-1} c_{n} \int_{Z_{i}-x}^{Z_{i+1}-x}\left[G\left(Z_{n+1}-x-y\right)\right.\right.$

$\left.\left.-G\left(Z_{n}-x-y\right)\right] \mathrm{d} G(y)\right\} \mathrm{d} G^{(j)}(x)$

$+\sum_{j=0}^{\infty} \bar{F}((j+1) T) \sum_{i=L}^{K-1} c_{i} \int_{0}^{Z_{1}}\left[G\left(Z_{i+1}-x\right)-G\left(Z_{i}-x\right)\right] \mathrm{d} G^{(j)}(x)$

$+c_{D}\left(\sum_{j=0}^{\infty} \bar{F}((j+1) T) \int_{0}^{Z_{1}} \bar{G}\left(Z_{K}-x\right) \mathrm{d} G^{(j)}(x)\right.$

$+\sum_{j=0}^{\infty} \bar{F}((j+2) T) \sum_{i=1}^{K-1} \int_{0}^{Z_{1}}\left[\int_{Z_{i}-x}^{Z_{i+1}-x} \bar{G}\left(Z_{K}-x-y\right) \mathrm{d} G(y)\right] \mathrm{d} G^{(j)}(x)$

$+\sum_{j=0}^{\infty} \bar{F}((j+2) T) \sum_{i=1}^{K-1} \int_{0}^{Z_{1}}\left[\int_{Z_{i-1}-x}^{Z_{i}-x} G\left(Z_{i+1}-x-y\right) \mathrm{d} G(y)\right] \mathrm{d} G^{(j)}(x)$

$+\sum_{j=0}^{\infty} \bar{F}((j+2) T) \sum_{i=1}^{K-1} \int_{0}^{Z_{1}}\left\{\sum_{n=i+1}^{K-1} \int_{Z_{i}-x}^{Z_{i+1}-x}\left[G\left(Z_{n+1}-x-y\right)\right.\right.$

$\left.\left.-G\left(Z_{n}-x-y\right)\right] \mathrm{d} G(y)\right\} \mathrm{d} G^{(j)}(x)$

$\left.+\sum_{j=0}^{\infty} \bar{F}((j+1) T) \sum_{i=L}^{K-1} \int_{0}^{Z_{1}}\left[G\left(Z_{i+1}-x\right)-G\left(Z_{i}-x\right)\right] \mathrm{d} G^{(j)}(x)\right)=c_{K}+\left(c_{D}-c_{K}\right) F(T)$

$+\sum_{j=0}^{\infty}\left[\left(c_{D}-c_{L}\right) \bar{F}((j+1) T)-\left(c_{D}-c_{K}\right) \bar{F}((j+2) T)\right] \int_{0}^{Z_{1}} G\left(Z_{L}-x\right) \mathrm{d} G^{(j)}(x)$

$-\sum_{j=0}^{\infty} \bar{F}((j+1) T) \sum_{i=L+1}^{K}\left(c_{i}-c_{i-1}\right) \int_{0}^{Z_{1}} G\left(Z_{i}-x\right) \mathrm{d} G^{(j)}(x)$

$-\sum_{j=0}^{\infty} \bar{F}((j+2) T) \sum_{i=2}^{L} \sum_{n=i}^{K}\left(c_{n}-c_{n-1}\right) \int_{0}^{Z_{1}}\left[\int_{Z_{i-1}-x}^{Z_{i}-x} G\left(Z_{n}-x-y\right) \mathrm{d} G(y)\right] \mathrm{d} G^{(j)}(x)$

Thus, the expected cost rate is, from (44) and (45), 
When $F(t)=1-\exp (-\lambda t)$ and $G(x)=1-\exp (-\mu x)$, (46) is:

$$
\begin{gathered}
c_{K}-\alpha \mathrm{e}^{\alpha \mu Z_{1}}\left\{\alpha \sum_{i=2}^{L} \sum_{j=i}^{K}\left(c_{j}-c_{j-1}\right)\right. \\
\times\left[\mathrm{e}^{\left.-\mu Z_{i-1}-\mathrm{e}^{-\mu Z_{i}}-\mu\left(Z_{i}-Z_{i-1}\right) \mathrm{e}^{-\mu Z_{j}}\right]}\right. \\
\frac{C_{6}\left(Z_{1}\right)}{\lambda}=\frac{\left.-\sum_{i=L+1}^{K}\left(c_{i}-c_{i-1}\right)\left(\mathrm{e}^{-\mu Z_{i}}-\mathrm{e}^{-\mu Z_{L}}\right)\right\}}{1-\alpha \mathrm{e}^{\alpha \mu Z_{1}}\left[\alpha \mathrm{e}^{-\mu Z_{1}}+(1-\alpha) \mathrm{e}^{-\mu Z_{L}}\right]}+c_{D}-c_{K}
\end{gathered}
$$

We find optimal $Z_{1}^{*}$ to minimize $C_{6}\left(Z_{1}\right)$. Differentiating $C_{6}\left(Z_{1}\right)$ with respect to $Z_{1}$ and putting it equal to zero,

$$
\begin{aligned}
& \frac{1-\alpha \mathrm{e}^{-(1-\alpha) \mu Z_{1}}}{1-\mathrm{e}^{-\mu\left(Z_{L}-Z_{1}\right)}}\left\{\sum_{i=2}^{L}\left(c_{i}-c_{i-1}\right)\left[1-\mathrm{e}^{-\mu\left(Z_{i}-Z_{1}\right)}+\frac{\alpha}{1-\alpha} \mu\left(Z_{i}-Z_{1}\right) \mathrm{e}^{-\mu\left(Z_{i}-Z_{1}\right)}\right]\right. \\
& \left.+\sum_{i=L+1}^{K}\left(c_{i}-c_{i-1}\right)\left[1-\mathrm{e}^{-\mu\left(Z_{L}-Z_{1}\right)}+\frac{\alpha}{1-\alpha} \mu\left(Z_{L}-Z_{1}\right) \mathrm{e}^{-\mu\left(Z_{i}-Z_{1}\right)}\right]\right\} \\
& +\alpha \mathrm{e}^{-(1-\alpha) \mu Z_{1}} \sum_{i=2}^{K}\left(c_{i}-c_{i-1}\right)\left[1-\mathrm{e}^{-\mu\left(Z_{i}-Z_{1}\right)}\right]=c_{K}
\end{aligned}
$$

When $L=K$, (48) is equivalent to (43).

\section{Numerical Calculation}

We assume that $G(x)=1-\exp (-\mu x), c_{i}=i(i=1,2,3, \ldots, K)$, and $Z_{i}=i(i=2,3,4, \ldots, K)$. Table 1 gives the optimal managerial cumulative damage $Z_{1}^{*}\left(0<Z_{1}^{*}<2\right)$ and the minimum cost rate $T C_{1}$ $\left(Z^{*}{ }_{1}\right)$ of Model 1 when $T=1, K=25,30,35$, and $\mu=1,1.5,2$. In this illustration, $Z^{*}{ }_{1}$ decreases and $T C_{1}\left(Z^{*}{ }_{1}\right)$ increases when $c_{K}$ and $1 / \mu$ increases.

Table 1. Optimal $Z^{*}{ }_{1}$ and $T C_{1}\left(Z^{*}{ }_{1}\right)$ of Model 1 for $K=25,30,35$ and $\mu=1,1.5,2$ when $T=1$.

\begin{tabular}{|c|c|c|c|}
\hline $\boldsymbol{c}_{\boldsymbol{K}}$ & $\boldsymbol{\mu}$ & $\boldsymbol{Z}^{\boldsymbol{*}_{\mathbf{1}}}$ & $\boldsymbol{T C}_{\mathbf{1}}\left(\boldsymbol{Z}_{\boldsymbol{1}} \mathbf{)}\right.$ \\
\hline \hline 25 & 1 & 1.444 & 1.385 \\
\hline \hline 30 & 1 & 1.387 & 1.442 \\
\hline 35 & 1 & 1.335 & 1.496 \\
\hline \hline 25 & 1.5 & 1.602 & 0.832 \\
\hline 25 & 2 & 1.657 & 0.604 \\
\hline
\end{tabular}


Table 2 presents the optimal managerial cumulative damage $Z^{*}$ and the minimum cost rate $T C_{2}\left(Z^{*}\right)$ of Model 2 when $T=1, K=25,30,35, \mu=1,1.5,2$. In this illustration, $Z^{*}{ }_{1}$ decreases and $T C_{2}\left(Z^{*}\right)$ increases when $c_{K}$ and $1 / \mu$ increases which are similar to Table $1 . Z^{*}{ }_{1}$ in Table 2 are smaller than those in Table 1.

Table 2. Optimal $Z_{1}^{*}$ and $T C_{2}\left(Z_{1}^{*}\right)$ of Model 2 for $K=25,30,35$ and $\mu=1,1.5,2$ when $T=1$.

\begin{tabular}{|c|c|c|c|}
\hline $\boldsymbol{c}_{\boldsymbol{K}}$ & $\boldsymbol{\mu}$ & $\boldsymbol{Z}_{\mathbf{1}}$ & $\boldsymbol{T C}_{\mathbf{2}}\left(\boldsymbol{Z}_{\mathbf{1}}\right)$ \\
\hline \hline 25 & 1 & 0.981 & 1.080 \\
\hline \hline 30 & 1 & 0.925 & 2.494 \\
\hline 35 & 1 & 0.924 & 2.822 \\
\hline \hline 25 & 1.5 & 0.981 & 0.884 \\
\hline 25 & 2 & 0.996 & 0.469 \\
\hline
\end{tabular}

Table 3 gives the optimal managerial cumulative damage $Z^{*}{ }_{1}$ and the minimum cost rate $T C_{3}\left(Z^{*}\right)$ of Model 3 when $T=1, L=10, K=25,30,35, \mu=1,1.5,2$. In this illustration, $Z^{*}$ decreases and $T C_{2}\left(Z^{*}{ }_{1}\right)$ increases when $c_{K}$ and $1 / \mu$ increases which are similar to Table 1 and 2 . $Z^{*}$ in Table 3 are smaller than those in Table 1 and 2.

Table 3. Optimal $Z^{*}$ and $T C_{3}\left(Z^{*}{ }_{1}\right)$ of Model 3 for $K=25,30,35$ and $\mu=1,1.5,2$ when $T=1$ and $L=10$.

\begin{tabular}{|c|c|c|c|}
\hline $\boldsymbol{c}_{\boldsymbol{K}}$ & $\boldsymbol{\mu}$ & $\boldsymbol{Z}_{\boldsymbol{*}_{1}}$ & $\boldsymbol{T C}_{\mathbf{3}}\left(\boldsymbol{Z}^{\left.\boldsymbol{*}_{1}\right)}\right.$ \\
\hline \hline 25 & 1 & 0.878 & 2.754 \\
\hline \hline 30 & 1 & 0.875 & 3.223 \\
\hline 35 & 1 & 0.873 & 3.691 \\
\hline \hline 25 & 1.5 & 0.973 & 0.992 \\
\hline 25 & 2 & 0.995 & 0.483 \\
\hline
\end{tabular}

We assume that $F(t)=1-\exp (-\lambda t)$. Table 4 presents the optimal managerial cumulative damage $Z_{1}^{*}$ and the minimum cost rate $T C_{4}\left(Z_{1}^{*}\right)$ of Model 4 when $T=1, K=25,30,35, \mu=1,1.5,2$, and $\lambda=0.1,0.02,0.01$. In this illustration, $Z^{*}{ }_{1}$ decreases and $T C_{4}\left(Z^{*}{ }_{1}\right)$ increases when $c_{K}$ and $1 / \mu$ increases which are similar to Table 1,2 , and $3 . Z^{*}$ and $T C_{4}\left(Z^{*}{ }_{1}\right)$ decrease when $\lambda$ decreases. $Z^{*}{ }_{1}$ and $T C_{4}\left(Z^{*}\right)$ in Table 4 are greater than those in Table 1.

Table 4. Optimal $Z^{*}$ and $T C_{4}\left(Z^{*}\right)$ of Model 4 for $K=25,30,35, \mu=1,1.5,2$, and $\lambda=0.1,0.02,0.01$ when $T=1$.

\begin{tabular}{|c|c|c|c|c|}
\hline $\boldsymbol{c}_{\boldsymbol{K}}$ & $\boldsymbol{\mu}$ & $\boldsymbol{\lambda}$ & $\boldsymbol{Z}^{\boldsymbol{*}}{ }_{1}$ & $\boldsymbol{T C}_{\boldsymbol{4}}\left(\mathbf{Z}^{\left.\boldsymbol{*}_{1}\right)}\right.$ \\
\hline \hline 25 & 1 & 0.1 & 1.548 & 11.260 \\
\hline \hline 30 & 1 & 0.1 & 1.487 & 11.316 \\
\hline 35 & 1 & 0.1 & 1.433 & 11.369 \\
\hline \hline 25 & 1.5 & 0.1 & 1.725 & 10.716 \\
\hline 25 & 2 & 0.1 & 1.762 & 10.502 \\
\hline \hline 25 & 1 & 0.02 & 1.465 & 3.359 \\
\hline 25 & 1 & 0.01 & 1.455 & 2.372 \\
\hline
\end{tabular}

Table 5 gives the optimal managerial cumulative damage $Z_{1}^{*}$ and the minimum cost rate $T C_{5}\left(Z_{1}^{*}\right)$ of Model 5 when $T=1, K=25,30,35, \mu=1,1.5,2$, and $\lambda=0.1,0.02,0.01$. In this illustration, $Z^{*} 1$ decreases and $T C_{5}\left(Z^{*}{ }_{1}\right)$ increases when $c_{K}$ and $1 / \mu$ increases which are similar to Table $1,2,3$, and 
4. $Z^{*}$ and $T C_{5}\left(Z^{*}{ }_{1}\right)$ decrease when $\lambda$ decreases which are similar to Table $4 . Z^{*}$ and $T C_{5}\left(Z^{*}{ }_{1}\right)$ in Table 5 are greater than those in Table 2 .

Table 5. Optimal $Z^{*}$ and $T C_{5}\left(Z^{*}\right)$ of Model 5 for $K=25,30,35, \mu=1,1.5,2$, and $\lambda=0.1,0.02,0.01$ when $T=1$.

\begin{tabular}{|c|c|c|c|c|}
\hline $\boldsymbol{c}_{\boldsymbol{K}}$ & $\boldsymbol{\mu}$ & $\boldsymbol{\lambda}$ & $\boldsymbol{Z}_{\boldsymbol{}_{\boldsymbol{1}}}$ & $\boldsymbol{T C}_{\boldsymbol{5}}\left(\boldsymbol{Z}^{\left.\boldsymbol{*}_{1}\right)}\right.$ \\
\hline \hline 25 & 1 & 0.1 & 1.458 & 11.709 \\
\hline \hline 30 & 1 & 0.1 & 1.272 & 11.870 \\
\hline 35 & 1 & 0.1 & 1.124 & 12.019 \\
\hline \hline 25 & 1.5 & 0.1 & 1.998 & 10.852 \\
\hline 25 & 2 & 0.1 & 1.999 & 10.555 \\
\hline \hline 25 & 1 & 0.02 & 1.329 & 3.913 \\
\hline 25 & 1 & 0.01 & 1.313 & 2.940 \\
\hline
\end{tabular}

Table 6 presents the optimal managerial cumulative damage $Z_{1}{ }_{1}$ and the minimum cost rate $T C_{6}$ $\left(Z_{1}^{*}\right)$ of Model 6 when $T=1, L=10, K=25,30,35, \mu=1,1.5,2$, and $\lambda=0.1,0.02,0.01$. In this illustration, $Z^{*}{ }_{1}$ decreases and $T C_{6}\left(Z^{*}\right)$ increases when $c_{K}$ and $1 / \mu$ increases which are similar to Table $1,2,3,4$, and $5 . Z^{*}$ and $T C_{6}\left(Z^{*}{ }_{1}\right)$ decrease when $\lambda$ decreases which are similar to Table 4,5 , and 6. $Z^{*}$ and $T C_{6}\left(Z^{*}{ }_{1}\right)$ in Table 6 are greater than those in Table 3.

Table 6. Optimal $Z^{*}{ }_{1}$ and $T C_{6}\left(Z^{*}{ }_{1}\right)$ of Model 6 for $K=25,30,35, \mu=1,1.5,2$, and $\lambda=0.1,0.02,0.01$ when $T=1$ and $L=10$.

\begin{tabular}{|c|c|c|c|c|}
\hline $\boldsymbol{c}_{\boldsymbol{K}}$ & $\boldsymbol{\mu}$ & $\boldsymbol{\lambda}$ & $\boldsymbol{Z}_{\boldsymbol{*}_{1}}$ & $\boldsymbol{T C}_{\boldsymbol{6}}\left(\mathbf{Z}_{\boldsymbol{1}}\right)$ \\
\hline \hline 25 & 1 & 0.1 & 1.252 & 11.834 \\
\hline \hline 30 & 1 & 0.1 & 1.078 & 12.010 \\
\hline 35 & 1 & 0.1 & 0.940 & 12.174 \\
\hline \hline 25 & 1.5 & 0.1 & 1.997 & 10.887 \\
\hline 25 & 2 & 0.1 & 1.999 & 10.561 \\
\hline \hline 25 & 1 & 0.02 & 1.138 & 4.045 \\
\hline 25 & 1 & 0.01 & 1.124 & 3.072 \\
\hline
\end{tabular}

Table 7 gives the optimal managerial cumulative damage $Z_{1}^{*}$ and the minimum cost rate $T C_{6}\left(Z_{1}^{*}\right)$ of Model 6 when $T=1, K=5, \mu=1, \lambda=0.1$, and $L=1,2,3,4,5$. In this illustration, $Z^{*}$ increases and $T C_{6}\left(Z^{*}\right)$ decreases when $L$ increases.

Table 7. Optimal $Z^{*}{ }_{1}$ and $T C_{6}\left(Z^{*}{ }_{1}\right)$ of Model 6 for $L=1,2,3,4,5$ when $T=1, K=5, \mu=1, \lambda=0.1$.

\begin{tabular}{|c|c|c|}
\hline $\boldsymbol{L}$ & $\boldsymbol{Z}_{\boldsymbol{1}}$ & $\boldsymbol{T C}_{\boldsymbol{6}}\left(\boldsymbol{Z}_{\boldsymbol{1}}\right)$ \\
\hline \hline 1 & 0.911 & 12.120 \\
\hline 2 & 0.976 & 12.057 \\
\hline 3 & 1.084 & 11.960 \\
\hline 4 & 1.165 & 11.897 \\
\hline 5 & 1.458 & 11.709 \\
\hline
\end{tabular}

The fluctuation tendencies of optimal managerial levels and their cost rates due to the fluctuations in parameters $c_{K}$ and $\mu$ are the same in Model 1,2,3, 4, 5, and 6, and are consistent with intuitive results. It is evident that optimal managerial levels of Model 2, 3, 5, and 6 which consider the maintenance delay, are smaller than those of Model 1 and 4 which do not consider the maintenance 
delay, because the maintenance delay raises the occurrence probability of CM. It is evident that cost rates of Models 4, 5 and 6 which consider the natural disaster are higher than those of Models 1, 2 and 3 which do not consider it. Managerial levels of Models 4, 5 and 6 are higher than those of Models 1, 2 and 3 because the risk of occurrence probability of CM due to natural disasters reduces the risk due to non-natural disasters. When $L$ increases, optimal managerial levels increase because the risk of occurrence probability of CM increases.

\section{Conclusions}

When the maintenance of infrastructure is planned, some unique characteristics which are different from mechanical ones must be considered. Because the scale of infrastructure is larger than that of mechanical systems, the relationship between damage and maintenance costs of infrastructure is complicated. Maintenances of some facilities must be postponed to the next fiscal year because the budget of local government is restricted. Because damages caused by natural disasters are inevitable, their effects must be considered when the maintenance plan is established. Based on a multiple cost model (Model 1) of Kishida et al. (2019) two expanded models (Model 2 and 3) which consider the maintenance delay have been considered. Furthermore, expanded models of these 3 models (Model 4, 5, and 6) which consider natural disasters have been established. We showed that there existed optimal solutions of Models 1 and 4 analytically and numerical calculation showed that there exist optimum values which minimizes expected cost rates of 6 Models. By performing sensitivity analysis of each Model, reasonable results could be obtained. By using these Models, the multiple managerial maintenance damage of infrastructure can be considered and the optimal maintenance can be clarified. These Models clarify which infrastructure should be prioritized under a limited budget. In particular, by considering the impact of natural disasters, these Models can be used effectively when requesting budgets to the central government and explaining to local residents. In the future, we plan to apply this maintenance model to an actual infrastructure system and confirm its usefulness.

\section{Conflict of Interest}

The authors confirm that there is no conflict of interest to declare for this publication.

\section{Acknowledgments}

The authors would like to thank the editor and anonymous reviewers for their comments that help improve the quality of this work.

\section{References}

Ito, K., \& Nakagawa, T. (2018b). Optimal maintenance policy of coastal protection systems. IEICE Technical Report, 118(365), R2018-45, 13-16.

Ito, K., Higuchi, Y., \& Nakagawa, T. (2018a). Optimal maintenance policy of social infrastructures. In Conference Proceedings, 24th ISSAT International Conference on Reliability and Quality in Design (pp. 2-4). Toronto, Ontario, Canada.

Kaito, K., Yasuda, K., Kobayashi, K., \& Owada, K. (2005). Optimal maintenance strategies of bridge components with an average cost minimizing principles. Proceedings of Journal of Japan Society of Civil Engineers, 801, 83-96. 
Kishida, T., Ito, K., Yoshiyuki, H., \& Nakagawa, T. (2019). Optimal maintenance models of social infrastructures considering natural disasters. In Conference Proceedings, 25th ISSAT International Conference on Reliability and Quality in Design (pp. 1-3). Las Vegas, Nevada, USA.

Kishida, T., Ito, K., Yoshiyuki, H., \& Nakagawa, T. (2020). Optimal maintenance models of social infrastructures considering natural disasters. In: Pham, H. (ed) Reliability and Statistical Computing Springer Series in Reliability Engineering. Springer, Cham, pp. 245-263.

Kobayashi, K., Eguchi, M., Oi, A., Aoki, K., \& Kaito, K. (2013). The optimal implementation policy for inspecting pavement with deterioration uncertainty. Journal of Japan Society of Civil Engineers, 1(1), 551- 568. DOI: 10.2208/journalofjsce.1.1_551.

Kobayashi, K., Eguchi, M., Oi, A., Aoki, K., Kaito, K., \& Matsumura, Y. (2012). The optimal repair and replacement model of pavement structure. Journal of Japan Society of Civil Engineers E1(Pavement Engineering), 68(2), 54-68.

Ministry of Land, Infrastructure, Transport and Tourism. (2017). White paper on land, infrastructure, transport and tourism in Japan. 114.

Nakagawa, T. (2007). Shock and damage models in reliability theory. Springer Verlag, London.

Obama, K., Kaito, K., Aoki, K., Kobayashi, K., \& Fukuda, T. (2012). The optimal scrapping and maintenance model of infrastructure considering deterioration process. Journal of Japan Society of Civil Engineers F4(Construction and Management), 68(3), 141-156.

Sakai, R., Onishi, Y., \& Otsu, H. (2014). The study of the maintenance management model of the tunnel structure using stochastic process. Proceedings of the Japan National Conference on Geotechnical Engineering, 39th, 2(2), 1711-1712.

Shin, H.C., \& Madanat, S. (2003). Development of a stochastic model of pavement distress initiation. $J$. Infrastructure Plan. and Man., Journal of Japan Society of Civil Engineers, 744(IV-61), 61- 67. DOI: https://doi.org/10.2208/jscej.2003.744_61.

Tsuda, Y., Kaito, K., Aoki, K., \& Kobayashi, K. (2005). Estimating Markovian transition probabilities for bridge deterioration forecasting. Structural Engineering/Earthquake Engineering, Journal of Japan Society of Civil Engineers, 801(73), 69-82.

Tsuda, Y., Kaito, K., Aoki, K., \& Kobayashi, K. (2006). Estimating Markovian transition probabilities for bridge deterioration forecasting. Structural Engineering/Earthquake Engineering, Journal of Japan Society of Civil Engineers, 23(2), 241s-256s. DOI: https://doi.org/10.2208/jsceseee.23.241s. 